\section{Properties of changing patterns evoking visually perceived oscillation*}

\author{
ERIK BÖRJESSON† \\ University of Uppsala, Uppsala, Sweden
}

The aim of this study was to identify some properties of changing proximal stimulus patterns which favor perceived oscillation. By using artificially generated stimulus patterns, it was found that the amount of changes associated with a certain direction of rotation should be small and the rate of these changes low if perceived oscillation was to appear. Great or swift changes were utilized by the visual system to determine perceived direction of rotation, and oscillation was not then reported. It was further found that patterns lacking straight edges perpendicular to the axis of rotation, or with this axis displaced from the middle of the pattern, and patterns with a gradient of texture density were perceived to oscillate more than similar patterns without these properties. Perceived oscillation of ellipses was discussed, and it was concluded that perceived oscillation was a consequence of perceived orientation, which is determined by stimulus properties.

Plane figures are sometimes perceived to change direction of rotation (oscillate) regularly although they are rotating in the same direction all the time. The best known example of this illusion is Ames"s trapezoidal window (Ames, 1951), although a variety of figures exhibit the effect (Pastore, 1952; Day \& Power. 1965). The explanation of the plienomenon commonly given states that the illusion is caused by misperceived orientation of the figure during half the revolution (Ames, 1951; Pastore, 1952; Graham, 1963).

Day and Power (1965), however, propose another interpretation. They state that misperceived orientation is a consequence of misperceived direction of rotation and not the other way around. Further, they stress the identity of proximal stimuli for two different orientations in depth as a necessary condition for apparent oscillation to appear. The perceived direction of rotation will then be determined by chance factors.

Two objections can be raised to Day and Power's general theory. First, two different orientations in depth never give identical proximal stimuli. The differences between proximal stimuli for clockwise and counterclockwise rotation have been discussed at some length by Jansson and Büriesson (1969a) and can be summarized in terms of changes at right angles to the axis of rotation (horizontal) and parallel to this axis (vertical). When rotating

*The author is indebted to Professor Gunnar Johansson and Dr. Gunnar Jansson for highly valuable discussions on this project, which was made possible by grants to Professor Johansson from The Swedish Council for Social Science Research and The Tricentennial fund of Bank of Sweden.

†Address: Department of Psychology. Svatbäcksgatan 10, S-75320 Uppsalu, Sweden. clockwise, the vertical edge of the figure moving toward the left is closer to the $S$, thus having a greater maxinum velocity proximally when passing the sagittal plane than the vertical edge moving toward the right. Concerning vertical changes, the parts of the figure to the right of the axis of rotation are approaching and thus growing proximally in the vertical dimension. For counterclockwise rotation. the opposite is true.

Hershberger (1967) discusses this weakness in Day and Power's theory and proposes that the theory is'valid when the difference between proximal stimuli for the two directions of rotation is subthreshold. However, in some cases oscillation is still perceived, although this difference is suprathreshold. The figure is than perceived to change shape (Jansson \& Böriesson, 1969b).

The other weakness in Day and Power's theory concerns factors affecting perceived motion. If the stimulus conditions are ambiguous, chance and motivational factors are beld to determine the perceived direction of rotation. This view, however. does not fit the fact that the illusion is very compelling with some figures although this is not the case with others. According to Day and Power, all figures should exhibit the illusion to the same extent, and for every figure perceived rotation is as probable as perceived oscillation.

The vicw advocated in the present paper can be summarized by two points. First, it is a necessary condition for apparent oscillation that proximal changes associated with a certain direction of rotation arc not utilized by the visual system to perceive rotation. It is predicted that these changes are not utilized if their amount or rate is decreased. Second, there are characteristics of the stimulus pattern which affect perceived orientation and consequently perceived motion of the pattern.

The aim of this study was to test these propositions and to identify some stimulus properties affecting perceived motion.

\section{AMOUNT AND RATE OF CHANGES ASSOCIATED WITH DIRECTION OF ROTATION}

In order to optimize the conditions for apparent oscillation, the changes associated with direction of rotation should not be utilized by the visual system. Usually, the amount of these changes is decreased by decreasing the visual angle of the stimulus pattern.

If a motion or a change occurs too slowly, it cannot be seen. One does not see the hour hand of a clock move. The speed of rotation of Ames's window is rather slow, between 3 and $6 \mathrm{rpm}$. Under these conditions it is probably more difficult for the visual system to utilize the changes associated with a certain direction of rotation than if the speed of rotation were swifter.

\section{EXPERIMENT 1}

The purpose of Experiment 1 was to study the effect of rate of change of a moving line on detection of that change.

\section{Method}

Stimuli. Each stimulus consisted of an artificially generated vertical line moving sinusoidally back and forth in the horizontal direction. When moving, the line sinusoidally clianged its length. The phase difference between the horizontal motion and the change of length was 90 or $270 \mathrm{deg}$. The line was projected onto a screen where the horizontal motion covered a distance of $28 \mathrm{~cm}$, corresponding to a visual angle of $33 \mathrm{deg}$. The mean length of the line was $11.7 \mathrm{~cm}$, corresponding to a visual angle of $14.2 \mathrm{deg}$. The horizontal motion and the change of length always had the same frequency, either 3 or $30 \mathrm{rpm}$ in the experiment.

Apparatus. The line was displayed by an oscilloscope (Tektronix 565). A line changing length was generated by a high-frequency voltage modulated by a sinusoidal low frequency fed into the $Y$ input of the oscilloscope. This low frequency also determined the horizontal back-and-forth motion of the line by being fed into the $X$ input. The phase difference (90 and $270 \mathrm{deg}$ ) between the horizontal motion and the change of length was obtained by using a variable phase function generator. The amount of clange of length could be varied by means of a potentiometer. The same method of generating stimuli has successfully been used by Johansson (1964). 
Table 1

Threshold Measures in Millimeters on the Screen

\begin{tabular}{llr}
\hline & \multicolumn{2}{c}{ Frequency of Line Motion } \\
\cline { 2 - 3 } Ss & $3 \mathrm{rpm}$ & $30 \mathrm{rpm}$ \\
\hline 1 & 20.75 & 5.50 \\
2 & 15.50 & 4.75 \\
3 & 10.75 & 5.00 \\
4 & 15.50 & 10.25 \\
5 & 30.00 & 7.00 \\
6 & 15.00 & 4.50 \\
Mean & 17.92 & 6.17 \\
\hline
\end{tabular}

Viewing conditions. The displayed line was projected onto a translucent screen by means of an optical device. The Ss were seated $67 \mathrm{~cm}$ in front of the screen. In order to minimize cues of two-dimensionality of the screen, a collimator lens was placed between the $S$ and the screen, giving parallel light rays. Leaning his chin on a support, the $S$ looked monocularly through the lens at a distance of $17 \mathrm{~cm}$. During the presentations, the room was darkened. Inspection time was free.

Procedure. The method of limits was used in the experiment. The line was first presented without any change of length. An increasing change of length was then introduced in discrete steps. For each step the difference between the maximum and the minimum length was increased by $2 \mathrm{~mm}$, and a report of what the $S$ perceived was requested. This procedure continued until two correct reports were given in succession. When the line reached its maximum length when moving toward the left (phase difference $=90 \mathrm{deg}$ ), the report was considered correct if it suggested a clockwise motion of the line in a circular or elliptical path. If the line reached its maximum when moving toward the right (phase difference $=270 \mathrm{deg}$ ), the motion would need to be seen as counterclockwise. Two measures for each frequency of line motion and phase difference were obtained, giving eight measures for each $\mathrm{S}$. The different conditions were presented in randomized order.

Subjects. Six undergraduate students of psychology served as Ss.

\section{Results}

In the beginning of a series, when the change of length was very small, the Ss always reported a line moving back and forth without changing length. When the change of length became greater and was utilized by the visual system, the Ss reported a line moving in a circular or elliptical path in depth. No reports were given of a line moving in a plane perpendicular to the line of sight changing length. Only three of hundreds of reports in the experiment indicated a perceived direction of motion not considered correct. In these cases the series was continued until the criterion was reached. In Table 1 the mean values of the threshold meaures for each $S$ and frequency are given. The numbers refer to the difference between maximum and minimum length of the line in millimeters on the screen. The mean length of the line was $117 \mathrm{~mm}$.

The data were $F$ tested. As the Ss were considered as a random variable, the error terms were chosen according to the mixed model (McGuigan, 1960). The result of the analysis is shown in Table 2 . The analysis revealed that all three sources were significant at a level of .01 .

\section{Conclusions}

Experiment 1 clearly shows that the rate of change affects perception of a line moving back and forth changing length. If the rate of change is decreased, a greater amount of change is necessary if it is to be utilized by the visual system.

\section{EXPERIMENT 2}

In order to evoke apparent oscillation, the conditions should be arranged to prevent the visual system from utilizing changes in proximal stimuli associated with a certain direction of rotation. It is predicted that this can be accomplished by decreasing the amount or rate of changes of the changing stimulus pattern. Earlier research has shown that the amount of change is a critical factor; increasing the amount of change makes the illusion disappear. However, no consistent effects of rate of change on apparent motion have been found (cf., for instance, Mulholland, 1956, and Zegers, 1964). The purpose of Experiment 2 was to study the effects of amount and rate of change on apparent motion of trapezoidal stimulus patterns. It was hypothesized that a decrease in amount or rate of change would favor apparent oscillation.

\section{Method}

Stimluli. The stimuli were outline oscilloscope-generated trapezoids changing shape to simulate rotating trapezoids. At its greatest width, each trapezoid covered a visual angle of $16 \mathrm{deg}$ and the longer and the shorter vertical edges covered angles of 16 and $8 \mathrm{deg}$, respectively. The stimulus patterns differed in three respects. First, they differed in rate of change between 3 and $30 \mathrm{rpm}$. Second, there were three different conditions of amount of change. The relative changes of length of the vertical edges were 1.07:1 (4 deg), 1.33:1 (16 deg), and 1.80:1 (32 deg). These three conditions differed in another respect concerning horizontal changes. Actually, both the vertical and horizontal changes were made to represent polar projections of rotating trapezoids seen under three different visual angles: 4, 16, and $32 \mathrm{deg}$ (the greater the visual angle, the greater the amount of change). However, the physical visual angle always remained $16 \mathrm{deg}$. Third, the phase relations between vertical and horizontal changes were 90 or $270 \mathrm{deg}$, representing clockwise and counterclockwise rotation. Combining these different variations makes 12 different stimulus patterns.

Apparatus and viewing conditions. The apparatus was the same as in Experiment 1 with one exception. The frequency generators were replaced by a digital computer (Linc-8) programmed to display the desired patterns. The viewing conditions were the same as in Experiment 1.

Procedure. Each stimulus pattern was presented once for each $S$ in randomized order. The $S$ was asked to report what kind of motion the pattern described and rate the rigidity of the pattern during this motion.

Subjects. Sixteen undergraduate students of psychology served as Ss.

\section{Results}

Oscillation or rotation was always perceived. In some cases a simultaneous translatory motion back and forth in depth was reported. In Table 3 frequencies of reported oscillation and rotation are summarized over Ss and phase relations. Thirty-two is the highest possible frequency for any treatment.

The effect of rate of change was tested with the Wilcoxon matched-pairs signed-ranks test (Siegel, 1956). The numbers of reported oscillations for the two conditions of rate of change were compared for each $\mathrm{S}$ who was thus considered as his own control. The obtained value of $\mathrm{T}=3$ is significant at a

Table 2

Analysis of Variance

\begin{tabular}{lcrrrr}
\hline $\begin{array}{c}\text { Source } \\
\text { of Variation }\end{array}$ & $\begin{array}{c}\text { Sum } \\
\text { of Square }\end{array}$ & df & \multicolumn{1}{c}{$\begin{array}{c}\text { Mean } \\
\text { Square }\end{array}$} & F & p \\
\hline lirequency & 1656.8 & 1 & 1656.8 & 18.9 & $<.01$ \\
Subjects & 558.9 & 5 & 111.8 & 8.3 & $<.01$ \\
F: S & 439.3 & 5 & 87.9 & 6.5 & $<.01$ \\
Within Groups & 487.0 & 36 & 13.5 & & \\
Total & 3142.0 & 47 & & & \\
\hline
\end{tabular}




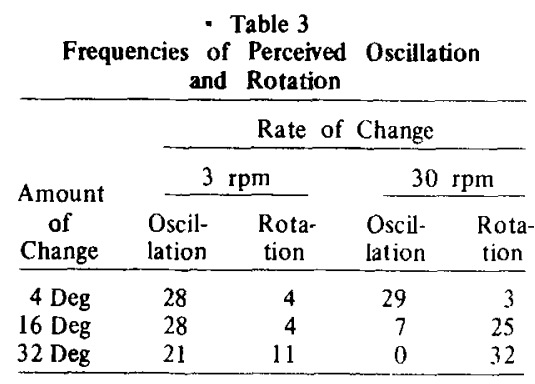

level of .005 , one-tailed testing for $N=16$

\section{Conclusions}

It is a necessary condition for apparent oscillation that the changes associated with a certain direction of rotation are not utilized by the visual system to determine that direction. This can be accomplished by decreasing the amount or rate of these changes.

\section{FIGURAL CHARACTERISTICS OF CHANGING PATTERNS \\ AFFECTING PERCEIVED MOTION}

Although amount and rate of change affect perceived motion, there are also figural characteristics of rotating figures which affect perceived motion. This view has received support in the literature. Thus it has been stated that the trapezoidal shape of Ames's window causes misperceived orientation and the refore the illusion occurs (Ames, 1951; Pastore, 1952: Graham, 1963).

\section{EXPERIMENT 3}

A trapezoidal window can be considered as consisting of two unparallel edges and two vertical edges of unequal length parallel to the axis of rotation. It is not clear to what extent each of these pairs of edges contributes to the illusion. A similar problem was studied by Power (1967) with quadrangular shapes. He found that shapes without horizontal edges were more often perceived to oscillate than were shapes with such edges. It was concluded that the change of horizontal edges (or rather, edges perpendicular to the axis of rotation) was utilized by the visual system to determine true direction of rotation, but that the change of vertical edges was not. The purpose of Experiment 3 was to study the relative contribution of unequal length of the vertical edges and convergence of the connecting edges on perceived uscillation.

\section{Method}

Stimuli. The stimuli were outlinc patterns. generated in the say way $a^{\text {c }}$ in Experiment 2. The stimuli continuously changed shape to simulate polar projections of rotating figures seen under a horizontal visual anglc of $16 \mathrm{deg}$, with the vertical axis of rotation in the middle of the figure. Thus there was a relative change of length of the vertical edges of $1.33: 1$. The stimulus patterns are shown in Fig. 1 as they appeared when having their greatest proximal width. For the $S$ they then covered a horizontal visual angle of $16 \mathrm{deg}$ and the longer-and the shorter-edge visual angles of 16 and $8 \mathrm{deg}$, respectively. Rate of change was $3 \mathrm{rpm}$.

Apparatus and viewing conditions. These were the same as in Experiment 2.

Procedure. Each S was presented each stimulus pattern once in randomized order and asked to report what kind of motion he perceived. The phase relation between horizontal and vertical changes, being 90 or $270 \mathrm{deg}$, simulating clockwise and counterclockwise direction of rotation, was balanced out in the experiment.

Subjects. Eight undergraduate students of psychology served as Ss.

\section{Results}

Only oscillation or rotation was reported. The number of Ss reporting oscillation is presented for each pattern in Fig. 1. After the experiment the Ss reported which end of the apparently oscillating patterns appeared closest to them. With few exceptions, the end with the greatest distance between the unparallel edges was perceived as closest, i.c., the right end of Patterns D, E, F. and $G$ in Fig. I. These patterns were generally perceived to oscillate, while patterns with parallel horizontal edges were perceived to rotate.

\section{Conclusions}

It is concluded that converging edges determine perceived orientation of the pattern regardless of the length of the vertical edges. Thus, patterns with straight edges perpendicular to the axis of rotation are correctly perceived 10 rotate, but

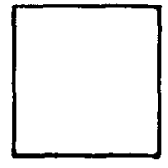

A 0

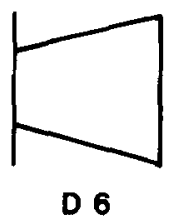

D 6

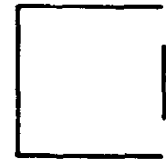

B 0

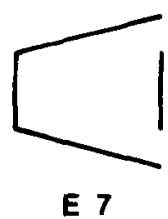

patterns with converging edges are perceived to oscillate with the end of the greatest distance between the converging edges closest to the $\mathrm{S}$. With the stimulus patterns used in Experiment 3 the vertical edges had no effect on perceived orientation. This result supports Power's (1967) conclusion that relative length change of edges parallel to the axis of rotation is ineffective as a cue to the direction of rotation. However, this conclusion should not be generalized to all shapes. Jansson and Börjesson (1969a) found, with stimulus patterns consisting of two vertical edges parallel to the axis of rotation without connecting edges, strong effects of relative length change.

\section{EXPERIMENT 4}

The absence of paraljel edges perpendicular to the axis of rotation seems to be a necessary condition for apparent oscillation. Converging edges are sufficient to evoke the illusion but not necessary. A variety of shapes without such edges are perceived to oscillate, including elliptical and irregular shapes. With Ames's trapezoidal window, the converging edges are sufficient for the illusion. However, the window has three more properties which miglıt affect perceived orientation and thereby favor perceived oscillation. First, shadows are painted to give cues of interposition. The effect of shadow-interposition cues on perceived motion has been studied by Cross and (ross (1969), who stress the importance of figural characteristics determining perceived orientation. They found that shapes with interposition cues more often were perceived to uscillate than were shapes without such cues. Second, panes are painted smaller toward the shorter vertical edge, giving a gradient of increasing texture density toward that end. The effect of a gradient of texture density on

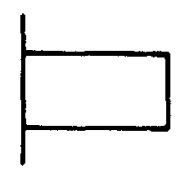

C 1
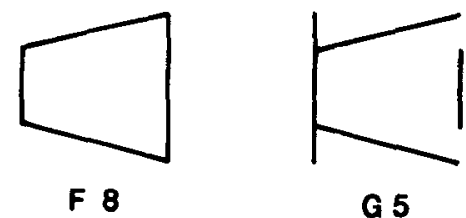

Fig. 1. Stimulus patterns in Experiment 3. Number of Ss reporting oscillation for each pattern is indicated. $\mathrm{N}=8$. 

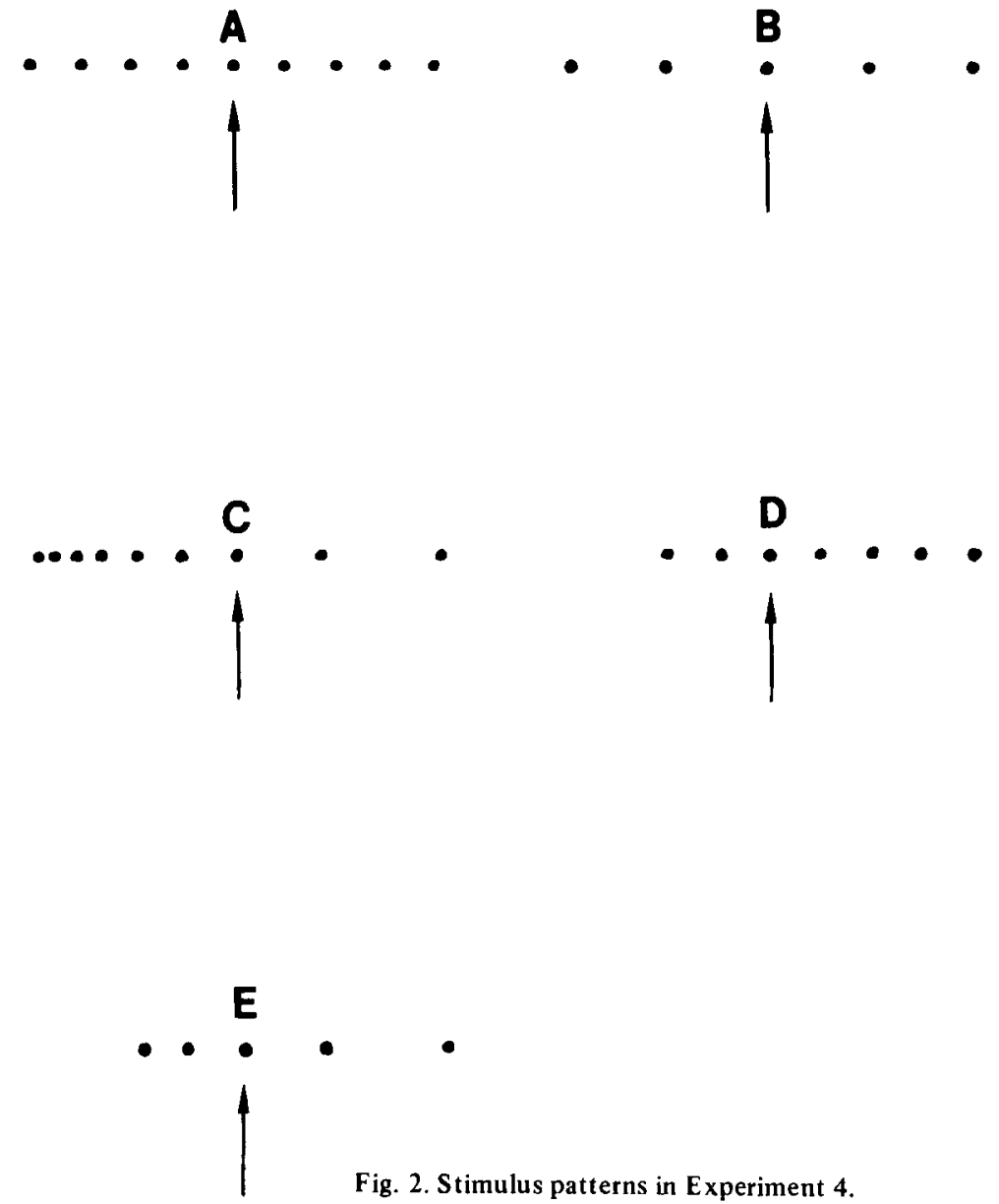

Fig. 2. Stimulus patterns in Experiment 4.

perceived slant is well known (Gibson, 1950). It is thus predicted that parts of a rotating figure with low texture density will be perceived as closer than parts with high density, and therefore the illusion will occur. Third, the axis of rotation is displaced from the middle of the figure toward the shorter vertical edge. This means that this edge will have a smaller proximal velocity than the end with greater distance to the axis of rotation. The effect of a gradient of proximal velocities on perceived slant has been studied by Gibson, Gibson, Smith, and Flock (1959), who found that greater velocities were perceived closer than were smaller velocities. It is consequently predicted that the end of a rotating figure toward which the axis of rotation is displaced and which has the smaller proximal velocity will be perceived as more distant, and that oscillation will be perceived. The aim of Experiment 4 was to test these predictions.

\section{Method}

Stimuli. The stimulus patterns consisted of oscilloscope-generated dots moving horizontally back and forth. In Fig. 2 the stimulus patterns are presented as they appeared when having their greatest width.
They then covered a visual angle of $16 \mathrm{deg}$, except Patterns D and E, which covered angles of $12 \mathrm{deg}$. The dots indicated by arrows were stationary and constituted an imagined axis of rotation. These arrows were not present in the experiment.

In Patterns A, B, and D the dots are evenly distributed. In Patterns $C$ and $E$ the dots are distributed to get a gradient of dot density. In Patterns D and E the imagined axis of rotation is displaced from the middle of the pattern. The predictions are confirmed if the right parts of Patterns $\mathrm{C}$, $D$, and $E$ in Fig. 2 are perceived as closer to the $S$ than the left parts and if the illusion is more probable for these patterns.

Eacli stimulus pattern was presented under three different conditions of relative proximal velocity of motion toward the right and the left, representing polar projections of rotation for three different visual angles: 4,16 , and $32 \mathrm{deg}$. The 4-deg angle involves a relative mean difference in proximal speed between motion to the left and to the right of an outermost dot of 1.05:1. Corresponding ratios for 16 and $32 \mathrm{deg}$ are $1.20: 1$ and $1.45: 1$. If the speed was greater for motion toward the left. a clockwise rotation was simulated; greater speed toward the right simulated a counterclockwise rotation. The frequency of the motion was $30 \mathrm{rpm}$.

Apparatus and viewing conditions. These were the same as in Experiment 2.

Procedure. Each stimulus pattern was presented three times with different relative velocities of motion toward the left and the right, which made 15 patterns for each $S$. These patterns were presented in randomized order, and the Ss had to report the kind of perceived motion. After the experiment the Ss had to report which end of Patterns C, D, and E appeared closest when oscillating. The effect of simulated direction of rotation was balanced out in the experiment.

Subjects. Sixteen undergraduate students of psychology served as Ss.

\section{Results and Conclusions}

Three Ss reported only rotation. For the remaining $13 \mathrm{Ss}$, the total numbers of perceived oscillation were $11,11,17,20$, and 19 for Stimulus Patterns A, B, C, D, and $E$, respectively. Although the effects were not very strong, the predicted Patterns C, D, and E obviously facilitated the illusion. Further, the Ss, with few exceptions, reported the longer end (Patterns D and E) and the end with lower dot density (Patterns $C$ and $E$ ) as appearing nearest when the pattern was perceived to oscillate.

\section{EXPERIMENT 5}

Experiment 5 reexamined the questions studied in Experiment 4 with other stimulus patterns, consisting of vertical lines instead of dots. It was supposed that line patterns would be more compelling than the dot patterns used in Experiment 4. The same predictions were made as in Experiment 4

\section{Method}

Stimuli and apparatus. A shadow-casting technique was used in this experiment. One of the three stimulus shapes shown in Fig. 3 was placed on a vertical axis between a punctiform light source and a translucent screen, the distance to the light source being $450 \mathrm{~cm}$ and to the screen, $16 \mathrm{~cm}$. The stimulus shapes consisted of nine vertical rods with a length of $17.5 \mathrm{~cm}$ and a diameter of $2 \mathrm{~mm}$. These rods were placed in a row between two horizontal bars at the upper and lower end of the rods. The widths of Shapes A, B, and C were 20,16 , and $22 \mathrm{~cm}$, respectively. A rectangle $(12 \times 28 \mathrm{~cm})$ was cut out of black pasteboard and mounted to the screen where the shadows were shown. Shadows of the horizontal bars were not visible in the rectangle. The speed of rotation was $12 \mathrm{rpm}$.

Viewing conditions and procedure. The 


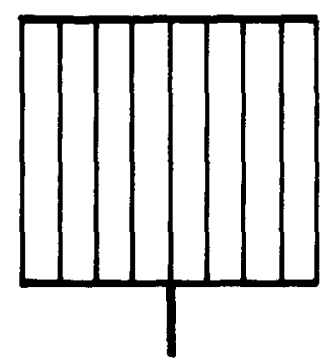

A 9

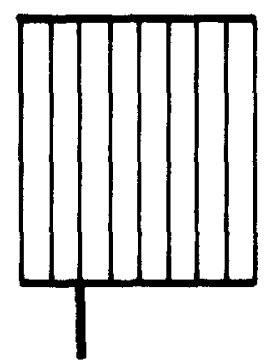

B 27

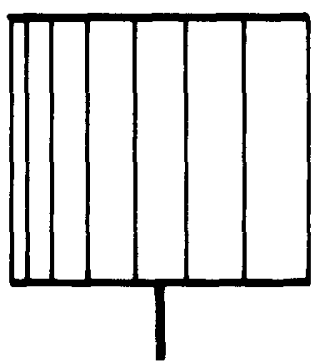

C 26
Fig. 3. Stimulus shapes in Experiment 5. The numbers of reported oscillations out of 38 are presented for each shape.

Ss were seated $55 \mathrm{~cm}$ in front of the screen and looked binocularly at the stimulus patterns. Shadows of each shape were presented for both clockwise and counterclockwise rotation in randomized order. The $S$ s reported perceived motion and in cases of oscillation pointed out the apparent nearest end of the pattern. Inspection time was free.

Subjects. Nineteen undergraduate students of psychology served as Ss.

\section{Results}

The numbers of reported oscillations for each shape are summarized over Ss and directions of rotation in Fig. 3. As each $S$ was shown each shape twice. he could get a score of 0,1 , or 2 , denoting number of reported oscillations for a certain shape. These data were used in testing the effect of shape with the Friedman two-way analysis of variance (Siegel, 1956). The obtained value of $\chi^{2}=8.71$ is significant at a level of .02 for $\mathrm{df}=2$. As predicted. Shapes B and C favored the illusion and Shape A did not. Further, the sliadows of the predicted ends of Shapes B and (' werc generally perceived as closest. Thus. nut of 27 reported oscillations, the end of Shape B, with more vertical rods, was perceived as closer in 21 cases. and out of 26 reported oscillations of Shape $\left({ }^{\circ}\right.$. Is were perceived with the end with low rod density as closest.

\section{Conclusions}

A gradient of rod density affects perceived orientation of a shape, the part with lower density all the time appearing closer, and therefore apparent oscillation occurs. It is probable that this conclusion holds also for other textures than those used in Experiments 4 and 5.

High velocities are perceived as closer than low and, therefore, the end with greater distance to the axis of rotation and higher proximal velocity is perceived as closer. Consequently, oscillation is perceived.

\section{DISCUSSION}

It is concluded that the trapezuidal window used by Ames has at least four characteristics that facilitate the illusion: the trapezoidal shape, the painted shadow-interposition cues, the texture gradient, and the eccentric axis of rotation. These characteristics usually give rise to misperceived orientation so that one edge is always perceived as farther than the other and therefore the apparent oscillating motion occurs.

There are other stimulus characteristics than those mentioned so far which aflect perceived orientation and motion. Mulholland (1958) found, with rotating T-shaped figures with a white disk at one end of the crossbar and a black disk at the other, that the disk appears to be oscillating in front, which has the greatest contrast with the background. Thus, perceived orientation can be affected by a reflectance gradient of the figure and therefore apparent oscillation is facilitated.

Day and Power $(1965)$ found, with stationary shapes, that the shorter vertical edge of rapezoids was alway's perceived as farther. even though it was objectively nearer. However, the general orientation of ellipses was correctly perceived. Since the two shapes when rotating exhibited about the same number of apparent reversals of direction, it was concluded that misperceived orientation is not a necessary condition for the occurrence of apparent uscillation. This conclusion does not necessarily follow from the evidence. On the contrary, the view advocated here states that apparent oscillation of ellipses is a consequence of misperceived orientation. A possible explanation for the occurrences of perceived oscillating cllipses is offered by a hypothesized perceptual tendency to perceive the ellipses as titted circles. If llis is truc. the ellipses will appear as tilted when being in the ironto-parallel plane. When the proximal width then begins to decrease. this is perceived as a change of dircction of rotation. This vicw is supported by evidence given by Day and Power $(1965)$. First. they found significant differences befween frequencies of reversals of direction for circles and ellipse's, the latter oscillating more. Second, a stationary ellipse in the fronto-parallel plane was judged to deviate $13.5 \mathrm{deg}$ from this plane, while a rectangular window deviated $2.4 \mathrm{deg}$.

Further evidence is given by Epstein, Jansson, and Johansson (1968) who studied the effect of the width-height ratio of rotating ellipses on perceived motion. They found that the frequency of oscillation reports was $2,7,9,10$, and 13 for the circle and the $.80, .60, .40$, and .20 ellipses, respectively. The median perceived angle of oscillation was $169,160,137,96$, and $82 \mathrm{deg}$ for the circle and the ellipses in order of decreasing width. The smaller the perceived angle of oscillation, the more probable is the occurrence of perceived oscillation. If the figure is perceived to oscillate with a small angle, it is perceived as being fur from the fronto-parallel plane orientation when it has its maximum proximal width and therefore the probability of perceiving rotation is small.

Effects of instruction, exposure time, and fixation point on perceived motion can, for the present, not be explained by the view advocated here. Concerning fixation point. however, a change in it can be considered as a change of proximal stimulus. If the point of fixation affects perceived orientation. it is predicted that perceived motion will be affected accordingly.

\section{REFERENCES}

AMES, A. Visual perception and the rotating trapezoidal window. Psychological Monographs, 1951, 65, No. 7.

CROSS. J.. \& CROSS, J. The misperception of rotary motion. Perception \& Psychophysics. $1969.5,94-96$.

DAY, R. H.. \& POWER, R. P. Apparent reversal fuscillation) of rotary motion in depth: An investigation and a general theory. Psychological Review. 1965, 72, 117-127.

EPSTEIN, W.. JANSSON, G.. \& JOHANSSON, (i. Perceived angle of oscillatory mution. Perception \& Piychophysics, 1968. 3, 12-16.

GIBSON. E. J., GIBSON, J. J.. SMITH, O. W., \& FLOCK. H. Motion parallax as a determinant of perceived depth. Journal of Experimental Psychology, 1959, 58,40-51.

GIBSON, J. J. The perception of the visual world. Bustom: Houghton Mifflin, 1950.

GRAHAM, C. H. On some aspects of real and apparent visuat movement. Journal of the Optical Sucicty of America. 1963. 53. $1019-1025$.

HFRSHBI:RG;ER, W. A. Comment on "Apparent reversial rescillation) of rotary motion in depth." Pychological Review, 1967.74. 235-238.

JANSSON. (i., \& BÖRJESSON, E. Percejved direction of rotary motion. Perception \& Psychophysics, 1969: 6, 19-26.

JANSSON. (i.. \& BÖRJESSON. E. Rotation and axillation as a function of imagined visual ingle and shape. In (i. Jansson (Ed.), Perceived rotary motion. Acta Universitas Upsaliensis. Abstracts of Uppsala Disscrtations in Social Sirences, 1969b. 2, 29-32.

JOHANSSON, G. Perception of motion and changing form. Scandinavian Journal of Piycholugy, 1964, 5. 181-208. 
McGUIGAN, F. J. Experimental psychology: $A$ methodological approach. Englewood Cliffs, N.J: Prentice-Hall, 1960.

MULHOLLAND, T. Motion perceived while viewing rotating stimulus-objects. American Journal of Psychology, 1956, 69, 96-99.

MULHOLLAND, $T$. The swinging disk illusion. American Journal of Psychology, 1958, 71 , 375-382.
PASTORE, N. Some remarks on the Ames oscillatory effect. Psychological Review, 1952, 59, 319-323.

POWER, R. P. Stimulus properties which reduce apparent reversal of rotating rectangular shapes. Journal of Experimental Psychology, $1967,73,595-599$.

SIEGEL, S. Nonparametric statistics for the behavioral sciences. New York: McGraw-Hill,
1956.

ZEGERS, R. T. The reversal illusion of the Ames' trapezoid. Transactions of the New York Academy of Sciences, 1964, 26, 377-400.

(Accepted for publication July 29, 1970.) 